\title{
FUCHS AND THE THEORY OF DIFFERENTIAL EQUATIONS
}

\author{
BY J. J. GRAY
}

Introduction. Lazarus Immanuel Fuchs (1833-1902) was the leading theorist of differential equations in the 1860s and 1870s, and, with Frobenius and Schwarz, a principal member of the 'second generation' of Berlin mathematicians. He obtained his Habilitation from Berlin University in 1865 and was eventually called back to succeed Kummer in 1884 . His work can profitably be seen as an attempt to impose upon the inchoate world of differential equations the conceptual order of the emerging theory of complex functions. As well as being the architect of the rigorous modern theory of linear equations, he raised many questions which were taken up by his contemporaries and provided an interesting battleground for the schools of invariant theory and transformation group theory. Most famously his work inspired Poincare to create the theory of Fuchsian functions and Fuchsian groups. Through his work and his career we can gain an insight into the state of mathematics in the second half of the nineteenth century, as the original work of Weierstrass was built up into an imposing intellectual edifice.

Differential equation theory before 1866 and Fuchs' first contribution. It took Fuchs some time to find his true area of interest as a mathematician. His doctoral thesis, written under Kummer's supervision, was on lines of curvature on a surface. Then he wrote two papers on number theory concerning the periods of the $n$th roots of unity when $n$ is not prime. But Kummer's influence was shortly to be outweighed by that of another. Weierstrass had been in Berlin as a professor at the Industrial Institute (the Gewerbeschule) since 1856, and in July 1864 he became professor at Berlin University. He had by then been lecturing at the University for some time. In the summer of 1863 he had lectured on Abelian functions and developed his theory of linear differential equations. This caught Fuchs' interest, and he then began to study the subject that occupied him for the rest of his life. His relationship with Weierstrass seems to have been close, and by 1870 he was writing to Casorati that he considered himself a pupil of Weierstrass (quoted in Neuenschwander [1978b, p. 46]).

Differential equations had been studied from various points of view by the 1860s, and we may distinguish two strands. One deals with particular equations, such as Legendre's equation (defined below) and its generalizations to

Received by the editors February 7, 1983.

1980 Mathematics Subject Classification. Primary 01A55; Secondary 01A70.

Key words and phrases. Ordinary differential equations, singular points, branch points, monodromy, elliptic functions, modular functions, Fuchsian functions, invariant theory. 
the hypergeometric equation and the differential equations underlying contemporary work on Abelian functions. The other sought to supply general existence proofs valid for large classes of differential equations. Legendre's equation (the name is due to Fuchs)

$$
k\left(1-k^{2}\right) \frac{d^{2} y}{d k^{2}}+\left(1-3 k^{2}\right) \frac{d y}{d k}-k y=0
$$

had been studied both by Legendre himself [1825] and Kummer [1836] because it describes the periods

$$
K=\int_{0}^{1} \frac{d x}{\left[\left(1-x^{2}\right)\left(1-k^{2} x^{2}\right)\right]^{1 / 2}}, \quad K^{\prime}=\int_{0}^{1} \frac{d x}{\left[\left(1-x^{2}\right)\left(1-k^{\prime 2} x^{2}\right)\right]^{1 / 2}}
$$

of the complete elliptic integrals as functions of the modulus $k$ (where $k^{a}+k^{\prime 2}=1$ ). Kummer recognized Legendre's equation as reducible to the hypergeometric equation

$$
x(1-x) \frac{d^{2} y}{d x^{2}}+[\gamma-(\alpha+\beta+1) x] \frac{d y}{d x}-\alpha \beta y=0,
$$

introduced and carefully studied by Gauss in his [1812a]. In fact, Kummer's study in some ways coincided with the then unpublished half of Gauss' paper [1812b]. The most important advances beyond Kummer's work were made by Riemann and Weierstrass in the 1850s. Riemann gave a thorough account of the hypergeometric equation from the standpoint of complex analysis in his paper [1857a] in which he introduced his $P$-functions. These are functions with three branch points on the complex sphere at which their branching behaviour is prescribed, and any three satisfy a linear relationship with constant coefficients. Riemann showed that from this information alone it is possible to deduce that the $P$-functions (with prescribed behaviour at the branch points) satisfy a hypergeometric equation whose coefficients can be determined. This accomplished two things: it showed that for this kind of equation the solutions determine the equation; and it clarified the nature of the solutions as functions of a complex variable. In particular, it clarified how they behave under analytic continuation, a matter on which Gauss had held perceptive views (not published until 1866), but which Kummer had avoided by confining his attention to equations with a real variable. Then, in an even more famous paper of 1857 [1857b] on the theory of Abelian functions, Riemann developed his theory of algebraic functions and their integrals. When he came to deal with Abel's theorem in \$\$14-16 of that paper, he discussed when a function can exist with prescribed zeros and poles by means of a system of linear differential equations. The same approach had been taken the year earlier by Weierstrass [1856] when dealing with the simpler case of hyperelliptic integrals, as Riemann acknowledged.

To understand Weierstrass' argument we may take the simpler case of elliptic integrals which he used as an illustration. The function $x=\operatorname{sn} u$, a Jacobian elliptic function, satisfies

$$
\frac{d^{2} \log x}{d u^{2}}=k^{2} x^{2}-\frac{1}{x^{2}} .
$$


Setting $x=p_{1} / p$ eventually yields two equations,

$$
\frac{d^{2} \log p_{1}}{d u^{2}}=\frac{-p^{2}}{p_{1}^{2}}, \quad \frac{d^{2} \log p}{d u^{2}}=\frac{-k^{2} p_{1}^{2}}{p^{2}},
$$

which, conversely, can be solved in terms of power series in $u$ which are uniformly convergent for all $u$. The arbitrary constants can be determined so that at $u=0$,

$$
p=1, \quad \frac{d p}{d u}=0, \quad p_{1}=0, \quad \frac{d p_{1}}{d u}=1
$$

when indeed $\operatorname{sn} u=p_{1} / p$. For the general theory Weierstrass had to consider $n$ linear ordinary differential equations in $n$ variables, corresponding to Jacobi's insight that one must invert sums of $n$ integrals each taken along $n$ paths. It is apparent that Weierstrass based his theory on differential equations and showed explicitly how the existence of solutions to such equations could be obtained as power series uniformly convergent on some domain. Unhappily, his lectures of 1863, which decisively influenced Fuchs, seem to be lost, but we do have a paper Weierstrass wrote in the 1840s (not published until 1894). In [1842] he studied the system of equations

$$
\frac{d x_{i}}{d t}=G_{i}\left(x_{1}, \ldots, x_{n}\right), \quad 1 \leqslant i \leqslant n,
$$

where the $G_{i}$ are rational functions, and showed how the $G_{i}$ can be modified so that a majorizing argument can be used to establish the uniform convergence of the power series which formally satisfy the original system of equations.

Weierstrass has led us to the question of general existence theorems for solutions to ordinary differential equations. The pioneer in these matters, as is well known, is Cauchy, chiefly in his [1835] in which he presented the method of majorants, but also in his [1840], which was less well known. In the 1850s the new generation of French mathematicians began to investigate the singularities of analytic functions; the crucial paper was the joint work of Briot and Bouquet [1856a] who were pupils of Liouville. They studied the equation $d y / d z=f(y, z)$ at points where the algebraic function $f$ is undetermined and showed that at such points the solutions may have branch points and poles, even essential singularities. As Neuenschwander has shown [1978a], Briot and Bouquet were unclear about the nature of essential singularities. In [1856b] they made a thorough study of those equations of the form $F(u, d u / d z)=0$, where $F$ is polynomial, which have elliptic functions as their solutions.

Fuchs based his first work on differential equations on the lectures of Weierstrass, Riemann's study of the hypergeometric equation, and the second study [1856b] of Briot and Bouquet. He published his findings in the Jahrsbericht of the Berlin Industry Institute [1865], and in a slightly revised form in Crelle's Journal [1866]. He argued that the main task was to find the properties of the solution to a differential equation at all points of the plane, in particular, whether the function is discontinuous or many-valued. By 'discontinuous' he meant what we mean by 'has a pole'. 
He considered the $\boldsymbol{n}$ th order linear ordinary differential equation

$$
\frac{d^{n} y}{d x^{n}}=p_{1} \frac{d^{n-1} y}{d x^{n-1}}+\cdots+p_{n} y
$$

whose coefficients $p_{i}$ are single-valued analytic functions everywhere on the complex sphere and have only finitely many poles, in short, are rational functions. He showed by a majorizing argument that away from the poles of the coefficients the equation has a basis of $n$ linearly independent solutions which can be specified by choosing the values of $y, d y / d x, \ldots, d^{n-1} y / d x^{n-1}$ at a nonsingular point. This observation was hardly new, and the route to the general form of the solutions was in any case soon considerably simplified by Frobenius [1873a]. But it implies, as Fuchs pointed out, that the singular points of the solutions must lie amongst the singular points of the coefficients, and so, in particular, they are fixed. Next he asked what restriction is to be placed on the coefficients if the solutions are not to have any 'bad' singularities, i.e., only finite poles and logarithmic branch points. He obtained a complete answer to this question, and here his results were new. (See Neuenschwander [1981] for a general discussion of the contemporary methods of complex function theory.)

The methods Fuchs employed derived from those of Riemann. He observed that if $\mathbf{y}=\left(y_{1}, \ldots, y_{n}\right)$ is an $n$-tuple of independent solutions valid in a neighbourhood of a singular point a of the equation, then analytic continuation of $\mathbf{y}$ around a returns it as another $n$-tuple of solutions, say $\tilde{\mathbf{y}}=\left(\tilde{y}_{1}, \ldots, \tilde{y}_{n}\right)$, and $\tilde{\mathbf{y}}=R \mathbf{y}$, where $R$ is an $n \times n$ matrix of constants. Replacing y by a different basis of solutions changes $R$ to a matrix of the form $B R B^{-1}$. In particular, taking $\mathbf{y}$ round every singular point in turn in the same direction takes $y$ along a contractible path on the sphere with the singular points removed, so Fuchs obtained an equation of the form

$$
R_{1} B_{2} R_{2} B_{2}^{-1} \cdots B_{\rho+1} R_{\rho+1} B_{\rho+1}^{-1}=I,
$$

whence $\operatorname{det}\left(R_{1} R_{2} \cdots R_{\rho+1}\right)=1$. Thus far, Fuchs had essentially followed Riemann, even to the extent of reproducing his notation. Now he went beyond. The eigenvalues of each $R_{i}$ are all that matter (Fuchs called them the roots of the fundamental determining equation). If $w_{1}$ is an eigenvalue corresponding to the solution $\mathbf{y}_{1}$ then $\tilde{y}_{1}=w_{1} y_{1}$, and $y_{1}$ is of the form $y_{1}=x^{r_{1}} \sum_{-m}^{\infty} a_{n} x^{n}$, where $w_{1}=e^{2 \pi i r_{1}}$. If $R_{i}$ can be completely diagonalized, say because its eigenvalues $w_{i 1}, \ldots, w_{i n}$ are all distinct, then the solutions are free of logarithmic terms and the corresponding $r_{i j}$ never coincide or differ by an integer. If, however, $R_{i}$ cannot be diagonalized, then logarithmic terms are to be expected. Fuchs' analysis fell just short of introducing the Jordan canonical form of a matrix, which was done by Jordan in [1870] and subsequently applied to differential equation theory by Hamburger [1873]. It preceded Weierstrass' study of canonical divisors [1868], which it may have influenced. (For a discussion of Weierstrass' work, see Hawkins [1977].) Fuchs also wrote down an equation for finding the $r_{i j}$ directly, which he called the fundamental equation, and which Cayley [1883] later successfully christened the indicial equation. But of greater significance was Fuchs' complicated passage from the matrices $R_{i}$ to a global study of the solutions, which culminated in his theorem that the linear ordinary 
differential equation (A) has all its solutions free of 'bad' singularities if and only if it is of the form

$$
\frac{d^{n} y}{d x^{n}}=\frac{F_{\rho-1}}{\psi} \frac{d^{n-1} y}{d x^{n-1}}+\frac{F_{2(\rho-1)}}{\psi^{2}} \frac{d^{n-2} y}{d x^{n-2}}+\cdots+\frac{F_{n(\rho-1)} y}{\psi^{n}},
$$

where $\psi=\left(x-a_{1}\right)\left(x-a_{2}\right) \cdots\left(x-a_{\rho}\right)$, and $F_{k}$ is a polynomial in $x$ of degree at most $k$. The equation has $\rho+1$ singular points: $a_{1}, a_{2}, \ldots, a_{\rho}$, and $a_{\rho+1}=\infty$. Moreover, there is one constraint to be satisfied by the indices $r_{i j}$ :

$$
\sum_{i=1}^{n} \sum_{j=1}^{\rho+1} r_{i j}=(\rho-1) \frac{n(n-1)}{2} .
$$

This derives from stipulated behaviour of the solutions.

As an example of his theory, Fuchs observed that the second-order equation of this type with two finite singular points $(\rho=2)$ is necessarily the hypergeometric equation. For such an equation, $F_{\rho-1}$ involves 2 free constants and $F_{2(\rho-1)}$ involves 3, so there are 5 free constants and 5 free indices $r_{i j}$. This enabled Fuchs to explain Riemann's result that the solutions determined the equation. For all other equations of order greater than 1 there are more constants available in the coefficients $F$ than in the set of indices $\left\{r_{i j}\right\}$, so the equation is not uniquely determined by the solutions. The extra coefficients have since become notorious as accessory parameters.

In conclusion, Fuchs asked what further restriction would have to be imposed on the coefficients before the solutions were all algebraic. This question was to attract a considerable amount of interest in the next decade, but at this stage Fuchs could only observe that the exponents at the singular points must be rational. All in all, the paper was a considerable achievement. It earned Fuchs his Habilitation, and Weierstrass said it showed that Fuchs "is completely familiar with the principles of the new function theory and is capable of independent research in the domain of analysis" (quoted in Biermann [1973, p. 94]).

Problems arising: notably, when are the solutions algebraic? Fuchs' work raised several questions for his colleagues to deal with. There was the question of what could usefully be said about equations which did not satisfy Fuchs' conditions, or were not, as came to be said, of the Fuchsian type. The pioneer in this area was L. W. Thomé who came to Berlin in 1863 and published a series of increasingly lengthy papers on the matter during the 1870 s. Since most of the named equations of mathematical physics (including Mathieu's, Bessel's, and Stokes') are not Fuchsian, and nor, for that matter, are the equations with constant coefficients of the Fuchsian type, the question is not an artificial one. Thomé made some progress, and his work was further simplified by Frobenius [1875], who had also contributed the idea of irreducibility of a differential equation [1873b]. He called an equation irreducible if it had no solutions in common with an equation of lower order, or of the same order but lower degree, and he showed that the hypergeometric equation is reducible if and only if one of its solutions is a polynomial. When Frobenius considered Thomé's work (Frobenius [1875]), he took up the case raised by 
Thomé [1872] when only one of the solutions in a basis has an essential singularity, and showed that in this case the indicial equation at a singular point is of order $n-1$ and the adjoint differential equation

$$
\frac{d^{n} y}{d x^{n}}=-\frac{d^{n-1}}{d x^{n-1}}\left(p_{1} y\right)+\cdots+(-1)^{n-1} \frac{d}{d x}\left(p_{n-1} y\right)+(-1)^{n} p_{n} y
$$

has a solution of the form

$$
\exp \left(c_{m} x^{-m}+\cdots+c x^{-1}\right) \sum_{r}^{\infty} a_{r} x^{\rho+r}
$$

However, Thomé's work did not shed much light on the nature of equations not of the Fuchsian type. One problem, that of exhibiting their solutions, is quite tricky, for the method of undetermined coefficients easily produces series with zero radius of convergence. Eventually, Poincare [1886] had the brilliant idea of interpreting these series as asymptotic solutions. This story is well told in Schlissel [1976] and will not be pursued further here.

A second question is what to make of the accessory parameters. By and large, despite some remarks by J. Thomae [1870] and Heun [1889], this problem proved too difficult. The best path forward was to consider equations which arise in specific ways that enable one to do something with them, and this was the path Fuchs himself took.

It was observed earlier that the periods of an elliptic integral, when considered as functions of the modulus, satisfy Legendre's equation. In [1870a] Fuchs looked at the periods of hyperelliptic integrals, considered as functions of a parameter. The paper is the most Riemannian of all his works, and in it he succeeded in showing that the periods satisfy an equation which is of the Fuchsian type. He was also able to recognize equations of this type amongst the equations satisfied by Abelian integrals [1871b] and by the theta null-values $\theta(0, \ldots, 0)$ as functions of the moduli [1871a]. Here he simplified some results of Thomae by basing his approach to the theta-functions on that of Clebsch and Gordan, in their Theorie der Abel'sche Functionen [1866]. These papers are interesting because they show a rising Berlin-school mathematician responding to Riemann's legacy, but it can hardly be argued that Fuchs did much to advance mathematics in them. He seems to have recognized this, for he soon abandoned the topic. He then wrote some papers seeking to simplify the study of the analytic continuation of multivalued functions before he returned to the problem he had raised in 1866: when are all the solutions to a differential equation algebraic?

He was recalled to the problem by a striking paper of Schwarz [1872], in which it was solved for the hypergeometric equation. Schwarz reduced the problem to the study of when the quotient of two independent solutions to a hypergeometric equation is algebraic. If the hypergeometric equation is

$$
\frac{d^{2} y}{d x^{2}}+p \frac{d y}{d x}+q y=0
$$


and two independent solutions are $f$ and $g$, then the quotient $f / g=\eta$ satisfies

$$
\Psi(\eta, x):=\left(\frac{d \eta}{d x}\right)^{-1} \frac{d^{3} \eta}{d x^{3}}-\frac{3}{2}\left(\frac{d^{2} \eta}{d x^{2}}\right)^{2}\left(\frac{d \eta}{d x}\right)^{-2}=2 p-\frac{1}{2} p^{2}-\frac{d p}{d x} .
$$

( $\Psi(\eta, x)$ is nowadays called the Schwarzian derivative, but it has a long history, and had been used by Kummer in his [1834], as Schwarz well knew.) Schwarz' first insight was to prove that this function maps the upper half-plane onto a circular-arc triangle whose angles $\lambda \pi, \mu \pi$, and $\nu \pi$ depend in a simple way on $\alpha, \beta$, and $\gamma$, the coefficients in $p$ and $q$. Then, under analytic continuation, it must map the lower half-plane onto a second, congruent, circular-arc triangle. When continued back into the upper half-plane, another triangle is produced, and so on. So if $\eta$ is to be algebraic, this process must cover the sphere with a finite number of these triangles. This is a strong restriction on $\alpha, \beta$, and $\gamma$, and Schwarz showed that the corresponding coverings of the sphere were the dihedrons, where the faces have angles $\pi / n$, $\pi / 2, \pi / 2$, and certain triangular subdivisions corresponding to the regular solids. Apart from the dihedral family, he found precisely 14 cases in which all the solutions to the hypergeometric equation were algebraic.

Schwarz then went on to investigate when the triangles produced a Euclidean tessellation of the plane; in this case $\eta$ is the inverse of an elliptic function, as he observed. Then, very significantly, he considered the case when the angle sum of each triangle is less than $\pi$, and he showed with the example of a triangle whose angles were $\pi / 3, \pi / 3, \pi / 5$, that in this case the triangles could fill out a finite disc. It then happens that the inverse function to $\eta$ is a single-valued function on the interior of the disc which has the boundary of the disc as a natural boundary, a phenomenon noticed earlier by Weierstrass in 1863. He gave this example of such a function, which he attributed to Kronecker:

$$
\sqrt{\frac{2 k}{\pi}}=1+2 q+2 q^{4}+2 q^{9}+\cdots
$$

No one responded for a while to the fertile new area that Schwarz discovered here, but his solution to the question of when the hypergeometric equation has algebraic solutions stimulated Fuchs to look again at his old problem. This time he formulated it in the language of invariant theory, which was a central topic of contemporary mathemetics. A homogeneous polynomial $f\left(x_{1}, \ldots, x_{n}\right)$ in $n$ variables is called a form in this language. Expressions in the coefficients which do not alter under linear transformations of the variables are called absolute invariants; expressions which only alter by some power of the determinant of the transformation are called relative invariants. Analogous expressions which involve the variables are called covariants. For example, the Hessian $\operatorname{det}\left(\partial^{2} f / \partial x_{i} \partial x_{j}\right)$ is a covariant, and if $g_{1}$ and $g_{2}$ are forms in 2 variables (i.e. binary forms) then their Jacobian $\operatorname{det}\left(\partial g_{i} / \partial x_{j}\right)$ is a simultaneous covariant. The whole thrust of invariant theory, especially as Gordan was then developing it, was the production of invariants and covariants of a given form. Gordan's main theorem [1868] was that there is a finite basis for the ring of all 
covariants of a binary form, although his proof was not very explicit. The theory of even ternary forms was still in a very primitive state.

Fuchs developed his analysis as follows [1876a]. He considered a basis of solutions only to a second-order differential equation, so that he could use the relatively well-developed theory of binary forms. Given such an equation, which can be written as

$$
\frac{d^{2} y}{d z^{2}}+q y=0
$$

he took binary forms $f\left(y_{1}, y_{2}\right)$ in the solutions $y_{1}$ and $y_{2}$, solutions which he supposed were algebraic functions with nonconstant quotient. In this case, every solution is algebraic. He then looked for binary forms of a particular kind, which he called ground forms. These were products

$$
\left(a_{1} y_{1}+a_{2} y_{2}\right) \cdots\left(a_{n 1} y_{1}+a_{n 2} y_{2}\right)
$$

which transformed under analytic continuation of $z$ only by being multiplied by a root of unity. Accordingly they are roots of rational functions. He showed that they exist and can be so obtained that $n$ is as small as possible, say $N$. They are characterized invariant-theoretically as the binary forms all of whose covariants of lower degree vanish, but Fuchs confessed in a letter to Hermite [1876b] that he was unable to solve the problem in this generality. Instead he approached it in an ad hoc way using a mixture of elementary invariant theory and the monodromy of the differential equation. Eventually he obtained a finite list of values for $N$ and the associated ground forms $\Phi\left(y_{1}, y_{2}\right)$, which culminated with these cases.

$$
\begin{array}{ll}
N & \Phi \\
4 & a_{0} y_{1}^{4}+a_{3} y_{1} y_{2}^{3} \\
6 & a_{1} y_{1}^{5} y_{2}+a_{3} y_{1}^{3} y_{2}^{3}+a_{5} y_{1} y_{2}^{5} \quad \text { or } \\
& a_{1} y_{1}^{5} y_{2}+a_{5} y_{1} y_{2}^{5} \\
8 & a_{1} y_{1}^{7} y_{2}+a_{4} y_{1}^{4} y_{2}^{4}+a_{7} y_{1} y_{2}^{7} \quad \text { (two cases) } \\
10 & a_{1} y_{1}^{9} y_{2}+a_{5} y_{1}^{5} y_{2}^{5}+a_{9} y_{1} y_{2}^{9} \\
12 & a_{1} y_{1}^{11} y_{2}+a_{6} y_{1}^{6} y_{2}^{6}+a_{11} y_{1} y_{2}^{11} \quad \text { (two cases). }
\end{array}
$$

Fuchs summarized his findings in two theorems. If the differential equation (B) has algebraic solutions, then either the general solution or the corresponding $\Phi$ is a root of a rational function, and, conversely, if a basis of solutions $\left\{y_{1}, y_{2}\right\}$ yields a form $\Phi\left(y_{1}, y_{2}\right)$ which is not of degree a power of 2 , then the differential equation has algebraic solutions. Recognizing that this is scarcely a simple test, he looked for simpler necessary conditions and found that unless $N=2$ it was necessary that no exponent at a singular point should have denominator greater than 10 . This condition, he observed, is indeed met by the exponents of those hypergeometric equations Schwarz had shown had only algebraic solutions.

Fuchs' work drew an immediate response from Felix Klein. While still at Erlangen in 1875, Klein had been told of Schwarz' work by Gordan, and 
studying it had greatly deepened his grasp of the mathematics of the icosahedron. On reading Fuchs' paper he saw at once that it contained superfluous cases, namely those $\Phi$ which do not describe the vertex net of a regular solid, and he commented to this effect in his Fortschritte review [1877, vol. IX, 172-173]. Klein's approach to the question of algebraic solutions was in fact much closer to that of Schwarz than Fuchs, as he was later to point out at length to Poincare when Fuchs' name became attached to Fuchsian groups.

Klein first gave a geometric proof [1875/76] that there are only finitely many finite groups of motions of the sphere, excluding the cyclic and dihedral families. Armed with this information he could then write down the form $f$ which specifies the vertex net of each regular solid, its Hessian $H(f)$ which locates the centres of the faces, and the Jacobian $T$ of $f$ and $H(f)$ which locates the mid-edge points. There is a relationship between $f, H(f)$, and $T$, as he showed, but which we shall not need. Then, in his [1876], he described the implications of the work for the problems of algebraic solutions. If a differential equation has algebraic solutions then its monodromy group $G$ is finite and therefore a known one of the list of possibilities. There is a canonical $G$-invariant rational function $Z=Z(z)$ associated to $G$ which is such that its inverse function $z=z(Z)$ satisfies the canonical Schwarzian equation in Schwarz' list: $\Psi(\eta, Z)=R(Z)$. If $y$ is a quotient of two solutions of the given differential equation, then its inverse function $\zeta=\zeta(y)$ is also $G$-invariant and rational, so $\zeta$ and $Z$ are rational functions of each other, so if $z=\phi(y)$ converts $Z=Z(z)$ into $Z(\phi(y))=\zeta(y)$, it converts the canonical equation into the given one, and the inverse transformation has the opposite effect. This elegant argument reduces the problem for the general second-order differential equation to the hypergeometric case. However, it is almost useless for solving the problem: characterize those second-order equations having only algebraic solutions. It deals rather better with the problem of determining whether or not a given equation has only algebraic solutions, a problem which has recently been rediscussed by Baldassarri and Dwork [1979].

Klein's solution certainly represented a victory for the new methods of group theory over the older ones of invariant theory, but the success was only partial because Klein emphasized the underlying geometry, as was his wont. But simultaneously, his friend Jordan gave an entirely group-theoretic solution which he was also able to extend to the third-order equation, and in some sense to the $n$th order. This was a dramatic illustration of the power of group theory.

The central insight recognized by Klein and Jordan was that a differential equation has only algebraic solutions if and only if its monodromy group is finite. Consideration of the Wronskian of two independent solutions of $d^{2} y / d z^{2}+p y=0$ shows that the monodromy group of this equation is a subgroup of $\operatorname{PSL}(2 ; \mathrm{C})$. Jordan classified these purely group-theoretically in [1876], using the newly available tools of Sylow theory. At first he made a trivial numerical mistake and lost the icosahedral group, but a letter from Klein put him right. Jordan dealt with the third-order case in the same way in the final section of the long paper he wrote for Klein's Mathematische Annalen [1878]. He had many more cases to consider by Sylow theory before the analogues of the groups of the regular solids were found in PSL( 3 ; C) and 
again he missed one, known most usually today as $\operatorname{PSL}(2 ; Z / 7 Z)$; see Gray [1982] for the story of Klein's discovery of it. Both men missed Valentiner's [1889] presentation of $A_{6}$ as a subgroup of $\operatorname{PSL}(3 ; \mathrm{C})$.

The $n$ th-order case led Jordan to one of his most famous theorems, the finiteness theorem, which asserts that in some sense $\operatorname{PSL}(n ; \mathrm{C})$ has only finitely many finite subgroups (see Jordan [1880]). The precise sense is not relevant to this discussion; but the case $n=2$ makes it clear that a preliminary classification into types of subgroups is needed since there are two infinite families of subgroups, the cyclic and dihedral ones. Then when $n=3$ one must take note of trivial cases of direct sums such as octahedral plus cyclic, and so on.

Jordan also considered what the related differential equations and their solutions would look like and proposed that only finitely many equations correspond to a given group, but Poincare [1884] later pointed out that this is incorrect because of the nature of the accessory parameters.

The study of third-order differential equations, all of whose solutions are algebraic, is necessarily more complicated, as Jordan's work had shown, and progress was much slower. In two papers [1882a, b] Fuchs showed that if the set of functions $\left\{y_{1}, y_{2}, y_{3}\right\}$ is a basis of solutions to a third-order equation and there is a homogeneous polynomial identity of order $n>2, f\left(y_{1}, y_{2}, y_{3}\right)=$ 0 , then the equation only has algebraic solutions. When $n=2$ the equation is satisfied by the squares of the solutions of a certain second-order equation; this class of third-order equations had earlier been studied by Brioschi [1879]. Fuchs also investigated the connection between the solutions of the differential equation and the genus $p$ of the Riemann surface defined by $f\left(y_{1}, y_{2}, y_{3}\right)=0$. Halphen's essay of 1881 published as [1884] extended this analysis to the $n$ th-order case. In awarding it the Grand Prix of the Academie des Sciences, Hermite said that it introduced the algebraic notion of invariants into the integral Calculus, and that the idea of the genus of an algebraic equation ("introduced into Analysis by Riemann and used so often in the works of our time") played an equally essential role in it.

By a happy coincidence, the essay that took second place was Poincaré's first significant work on Fuchsian functions. Whereas Halphen's approach is a high water mark for the methods of invariant theory, the work of Jordan and Poincaré helped open up a fertile area for the newer methods of group theory, and so displayed these methods to advantage in the study of problems in a central topic in contemporary mathematics. Poincare's essay derived from another new departure by Fuchs, to whom we must now return, although not immediately to the work that inspired Poincaré.

Elliptic and modular functions; the arrival of Poincaré. In a geometrical sense, the ordinary differential equations which lie immediately beyond the equations with rational functions as coefficients are those with elliptic functions as coefficients. Of these, the simplest and most historically and physically significant is Lamé's equation. It can be written variously as

$$
\frac{d^{2} y}{d u^{2}}-(n(n+1) \wp(u)+B) y=0,
$$


using the Weierstrassian elliptic function $\wp(u)$, or as

$$
\begin{gathered}
\frac{d^{2} y}{d x^{2}}-\frac{1}{2}\left[\frac{1}{x-e_{1}}+\frac{1}{x-e_{2}}+\frac{1}{x-e_{3}}\right] \frac{d y}{d x} \\
-\frac{(n(n+1)+B) y}{4\left(x-e_{1}\right)\left(x-e_{2}\right)\left(x-e_{3}\right)}=0,
\end{gathered}
$$

which exhibits it as an equation of the Fuchsian type with four singular points. As such, it is the simplest example of Heun's equation, in which the accessory parameter is an integer $n$. It may also be written in terms of the Jacobian elliptic function sn $v$. Lamé introduced it in the first form in his [1845], and Halphen studied it in the second form by writing $x=\wp(u)$ in his [1888]. In the third form it was studied by Hermite [1877] and Fuchs [1878a].

In a series of papers written between 1877 and 1882, Hermite showed that the solutions to Lamé's equation were what Picard was soon to call elliptic functions of the second kind. These are functions $F$ for which there are two quasi-periods, $2 K$ and $2 i K^{\prime}$ :

$$
F(x+2 K)=\mu F(x), \quad F\left(x+2 i K^{\prime}\right)=\mu^{\prime} F(x),
$$

for some constants $\mu$ and $\mu^{\prime}$. Hermite's work begins a considerable flurry of activity aimed at showing how elliptic functions of both the old and new kinds could be used in applied mathematics.

Fuchs showed first of all, in his [1877c] and [1878a], how Hermite's work related to his own, and then in his $[\mathbf{1 8 7 8 c ]}$ he asked what conditions must be imposed on $P$ if the differential equation $d^{2} y / d x^{2}=P y$ has a basis of solutions consisting of two elliptic functions of the second kind whose poles are the poles of $P$. He showed that it was necessary that $P$ be a single-valued doubly-periodic function of $x$. Picard then showed in his [1879, 1880a, b] that this condition is in general sufficient; the anomalous cases were treated by Mittag-Leffler [1880]. The consummation of this line of enquiry was Halphen's prize-winning essay of 1881 already mentioned.

By now Fuchs had moved on to a more general question, which is the one that caught Poincarés interest. Fuchs took the general second-order differential equation of the Fuchsian type,

$$
\frac{d^{2} y}{d z^{2}}+P(z) \frac{d y}{d z}+Q(z) y=0
$$

$P$ and $Q$ are rational functions, and supposed $f_{1}$ and $f_{2}$ were linearly independent solutions of it. He then asked the following question, which is a naive generalization of the Jacobi inversion problem. Consider

$$
\begin{aligned}
& \int_{\zeta_{1}}^{z_{1}} f_{1}(z) d z+\int_{\zeta_{2}}^{z_{2}} f_{1}(z) d z=u_{1}\left(z_{1}, z_{2}\right), \\
& \int_{\zeta_{1}}^{z_{1}} f_{2}(z) d z+\int_{\zeta_{2}}^{z_{2}} f_{2}(z) d z=u_{2}\left(z_{1}, z_{2}\right),
\end{aligned}
$$


and ask: what can be said about $z_{1}$ and $z_{2}$ as functions of $u_{1}$ and $u_{2}$ ? He sometimes imposed an auxiliary condition which required the inverse function $z=z(\zeta)$ to be single-valued if $\zeta=f_{1}(z) / f_{2}(z)$.

It cannot be said that the eight papers Fuchs wrote on this subject were a great success. Poincare, indeed, took as his starting point for his prize essay the observation that Fuchs' case-by-case analysis was both confusing and incomplete. It certainly confused Fuchs, because some of his results contradicted ones he had earlier obtained in his study of the problem of when a differential equation has all its solutions algebraic. In the correspondence of 1880 between Poincaré and Fuchs (published in, e.g., Poincaré, Oeuvres, XI, pp. 13-25) Poincaré even had to explain how to distinguish between single-valued and unbranched functions. The nub of the problem can be indicated by considering what for Fuchs was just the auxiliary condition that $z=z(\zeta)$ be single-valued, but which Poincaré rightly emphasized was the central problem. Suppose, for simplicity, the differential equation has three singular points and so is the hypergeometric equation. Then, if $\gamma$ is a loop based at $z_{0}$ and enclosing a singular point, analytic continuation of $f_{1}$ and $f_{2}$ around $\gamma$ returns them as, say, $a_{1} f_{1}+a_{2} f_{2}$ and $b_{1} f_{1}+b_{2} f_{2}$, respectively. The variable $z$ has returned to $z_{0}$, so the quotient $f_{1} / f_{2}$ satisfies

$$
\frac{f_{1}\left(z_{0}\right)}{f_{2}\left(z_{0}\right)}=\frac{a_{1} f_{1}\left(z_{0}\right)+a_{2} f_{2}\left(z_{0}\right)}{b_{1} f_{1}\left(z_{0}\right)+b_{2} f_{2}\left(z_{0}\right)} .
$$

Consequently, the inverse function is invariant:

$$
z_{0}\left(f_{1} / f_{2}\right)=z_{0}\left(\frac{a_{1} f_{1} / f_{2}+a_{2}}{b_{1} f_{2} / f_{2}+b_{2}}\right)=z_{0} .
$$

The function $f_{1} / f_{2}$ is holomorphic everywhere except at the three singular points of the differential equation, which may be put at $z=0,1$ and $\infty$ if need be by a rational change of variable. So $f_{1} / f_{2}$ maps the upper half-plane onto a circular-arc triangle and, under analytic continuation maps the Riemann sphere onto a net of triangles, as Schwarz had shown. The inverse function will therefore be single valued provided that the net of triangles does not overlap. Fuchs' work produced conditions sufficient to guarantee that his functions $z_{1}=z_{1}\left(u_{1}, u_{2}\right)$ and $z_{2}=z_{2}\left(u_{1}, u_{2}\right)$ were holomorphic, but Poincaré pointed out that this only showed that $z=z\left(f_{1} / f_{2}\right)$ was not branched, not that the function was single valued. It left open the possibility that the net of triangles filled out, for example, an annular region. Eventually Fuchs pushed his results to a complete solution, given in his [1887d]. An amusing conclusion of his is that the original differential equation cannot have more than 6 finite singular points.

It seems that Fuchs' lack of geometrical grasp may have let him down. In an earlier paper [1877b] he dealt with this question of Hermite: why is it that if $K$ and $K^{\prime}$ are complete elliptic integrals, and so solutions of Legendre's equation, and one defines $H=K / K^{\prime}, q=e^{-\pi H}$, then this, as a function of $q$, has the unit circle as a natural boundary, but the same is not true if $K$ and $K^{\prime}$ are replaced by $J$ and $J^{\prime}$, Legendre's complete elliptic integrals of the second kind. Fuchs had answered this question by comparing the monodromy matrices in 
each case. When $K$ and $K^{\prime}$ are considered, the monodromy matrices are of the form

$$
\left(\begin{array}{cc}
a & 2 b i \\
2 c i & d
\end{array}\right)
$$

where $a, b, c$, and $d$ are integers. So under arbitrary analytic continuation the general form of $H$ is known, and from it Fuchs could deduce the behaviour of $q$. He showed that the inverse function takes the values 0,1 and $\infty$ on the circle $|q|=1$ (although he got their distribution wrong as Dedekind speedily showed in his [1877]). Then he showed that the corresponding story for $J$ and $J^{\prime}$, starting from the differential equation which they satisfy, is suitably different.

Hermite was very pleased with the result, which enabled him to avoid using Riemann's methods. In fact the question is a deep one. Fuchs had shown what seems to have been known to Gauss by 1805 (see Gauss, Werke, III, p. 477) and Riemann (see his Werke, 2nd edition, 1892, p. 455 for Dedekind's analysis of a Riemannian fragment), namely that $k^{2}=k^{2}\left(K / K^{\prime}\right)$, the elliptic modular function, is invariant under transformations $z \rightarrow(a z+2 b i) /(2 c i z+d)$. Allowing for a rotation of the complex plane by $\pi$, which amounts to replacing $K$ by $i K$, this is saying that $k^{2}$ is invariant under the subgroup of $\operatorname{SL}(2: \mathbf{Z})$ which is the kernel of the map of matrices induced from the mapping $\mathbf{Z} \rightarrow \mathbf{Z} / 2 \mathbf{Z}$. Fuchs did not express his result this way, preferring his monodromy matrices and the quotient $K / K^{\prime}$, but Hermite in his reply stressed the invariance of the inverse function. Unlike Gauss and Riemann, neither drew a picture of the fundamental domain for $k^{2}$ (Fuchs did so in a much later paper [1893], by which time the material was quite generally known). The first to stress the group-theoretical aspect seems to have been Klein in his [1878/79] (see Gray [1982]).

It seems worth mentioning that Fuchs' attack on the problem was to replace the parameter $k^{2}$ in the complete elliptic integrals $K$ and $K^{\prime}$ by $\lambda^{-1}$. This quadratic transformation $k^{2}=\lambda$, as it is called, reduces Legendre's equation to the hypergeometric equation satisfied by $K \sqrt{ } \lambda$ and $K^{\prime} \sqrt{ } \lambda$, where $K$ and $K^{\prime}$ are now regarded as functions of $\lambda$. This equation,

$$
2 \lambda(\lambda-1) \frac{d^{2} y}{d \lambda^{2}}+2(2 \lambda-1) \frac{d y}{d \lambda}+\frac{1}{2} y=0
$$

has since become known as the Picard-Fuchs equation. It has a modern significance in algebraic geometry which is beautifully explained in Clemens [1980].

At all events, Fuchs did indeed answer his original question. I have attempted a detailed exposition of his work in my [1983b] and will not repeat the attempt here. The vital response was Poincaré's. Poincaré, as has been said, considered only the question about the inverse to the quotient of two solutions to a differential equation. We have two accounts of his progress. One is his celebrated essay [1908] in which he emphasized the psychological aspects of his discoveries. The other comprises the three unpublished Suppléments to his essay, recently discovered and described in Gray [1981, 1983a]. Taken together they show almost week by week how the hypergeometric equation was studied 
by Poincare until the realization came (boarding a bus at Coutances) that when the triangles fill out a disc analytic continuation moves them around by non-Euclidean rigid-body motions. This realization dominates the first supplement, and it enabled him to prove the convergence of certain holomorphic quasi-invariant functions, which he called theta-Fuchsians, whose quotients represented the inverse function $z=z\left(f_{1} / f_{2}\right)$. A second unexpected appearance of non-Euclidean geometry when he was pondering the number theory of $a^{2}+b^{2}-c^{2}$ enabled Poincare to escape the hypergeometric case and turn to the general second-order equation, as the second supplement describes. An unrigorous continuity method then enabled him in the third supplement to deal with Legendre's equation, when the circular-arc triangles have zero angles.

These papers are the start of Poincarés Fuchsian and Kleinian theories (see his [1882a, b, 1884, etc.]). It is well known that Klein hotly contested the appropriateness of calling them after Fuchs, and indeed Poincaré admitted (27 June 1881) that had he known of Schwarz' work he would have called them after Schwarz instead. But something in Klein's ferocity must have annoyed Poincaré, because he christened related objects Kleinian and observed in German that "Name is Sound and Fury" (4 April 1882). The letters between the two men make fascinating reading and have often been reprinted, for example in Klein's Gesammelte Mathematische Abhandlungen, III, 1923, pp. 587-621, and in Poincaré's Oeuvres, XI, 1953, pp. 26-65. The controversy became public in December 1881 when Klein added editorial comments to the paper he had commissioned from Poincare for the Mathematische Annalen, and Fuchs replied with a carefully written note in the Göttingen Nachrichten [1882a]. However the question of influence may be resolved, it is not exactly true that Klein began his work on functions "which remain unchanged under linear substitutions" as a result of Fuchs' work, which was Fuchs' final claim. As we have seen, Klein was already interested in such a question when Fuchs returned to the algebraic solutions question.

Fuchs' work in the 1880s. The year 1884 marks in some ways the last high point of Fuchs' career. He received the final accolade of a full professorship at the University of Berlin and was made a full member of the Berlin Academy of Sciences. He also solved a question suggested by his earliest work on differential equations: characterize those (nonlinear) equations which have only fixed branched points none of which depend on the initial conditions. He considered in his [1884a] only first-order differential equations $F\left(x, y, y^{\prime}\right)=0$, where $F$ is a polynomial in $y$ and $y^{\prime}$ whose coefficients are single-valued functions of $z$, and showed by relatively elementary considerations that the necessary and sufficient conditions for the equation

$$
F\left(z, y, y^{\prime}\right)=a_{0} y^{\prime m}+a_{1} y^{\prime m-1}+\cdots+a_{m}=0
$$

to have only fixed branch points, where the $a$ 's are polynomials in $y$ whose coefficients are rational functions of $z$, were:

1. $a_{0}$ is independent of $y$, and $a_{k}$ is of degree at most $2 k$ in $y$; 
2. if $y=\eta$ is a root of the discriminant $D(z, y)=0$ of $F$, for which $y^{\prime}$ is branched as a function of $y$, then $\eta$ also satisfies the differential equation, and the values of $y^{\prime}$ lying over $y=\eta$ are all $y^{\prime}=d \eta / d z$;

3. if the root $\eta^{\prime}=d \eta / d z$ is of multiplicity $\alpha$ then $\eta$ is a root of $F\left(z, y, y^{\prime}\right)=0$ of multiplicity at least $\alpha-1$.

[The discriminant is obtained by eliminating $y^{\prime}$ from $F\left(z, y, y^{\prime}\right)=0$ and $\partial F / \partial y^{\prime}=0$; it picks out multiple points on the curve $F=0$.]

Condition (1) derives from stipulating that $d y / d z$ be finite when $z=\infty$. This is readily seen in the case of first degree equations:

$$
a_{0}(y) y^{\prime}+a_{1}(y)=0 .
$$

If one sets $\tilde{y}=1 / y$, then the equation becomes

$$
-\tilde{a}_{0}(\tilde{y}) \frac{d \tilde{y}}{d z}+\tilde{y}^{2} \tilde{a}_{1}(\tilde{y})=0 .
$$

To avoid the situation where an arbitrary choice of $(z, \tilde{y})=(\alpha, 0)$ makes $d \tilde{y} / d z$ infinite, it is necessary that $\tilde{y}^{2} \tilde{a}_{1}(\tilde{y})$ be finite when $\tilde{y}=0$. So $\tilde{a}_{1}$ must be of degree at most 2 in $\tilde{y}$, and $a_{1}$, consequently of degree at most -2 in $y$. This shows that the most general first-order and first degree equation with only fixed branch points is

$$
\frac{d y}{d z}=g_{0}(z)+g_{1}(z) y+g_{2}(z) y^{2}
$$

the famous Riccati equation. For equations of higher degree the corresponding result is obtained by using suitably chosen local coordinates.

Fuchs then enquired about the special cases which arise when the genus of the curve relating $y$ and $y^{\prime}$ is 0 or 1 , cases which arise, he showed, when the factors of the discriminant $D(y)=0$, which give the branch points of $y^{\prime}$ at $y=\eta$, are independent of $z$. He showed that when $p=0$ the equation is rationally transformable to Riccati's equation; the case $p=1$ was shown to reduce to

$$
\frac{d t}{d z}=A_{0}+A_{1} t+A_{2} t^{2}+\lambda \sqrt{ }(R)
$$

where $A_{1}, A_{2}, A_{3}$ and $\lambda$ are functions of $z$ alone, and $R$ is a polynomial of degree 4. This result followed almost at once from Clebsch's form for a rational function on a surface of genus 1 :

$$
y=\frac{\phi_{1}+\psi_{1} / R(t)}{\phi_{0}+\psi_{0} / R(t)},
$$

where the $\phi$ 's and $\psi$ 's are polynomials in $t$ of degree $\leqslant m / 2$ (Clebsch [1864, p. 222]).

Fuchs failed to discuss the cases where $\omega \rightarrow \infty$ as $z \rightarrow z_{0}$ with complete thoroughness. The necessary modifications to his theorem are clearly described in Ince [1926, p. 311], whose discussions in Chapters 13 and 14 are still the best introduction to this part of the theory of differential equations. Matsuda [1980] also notes that Fuchs missed a point here, in his account of first-order algebraic differential equations from the standpoint of modern differential 
algebra. The example, due to Hill and Berry, which shows that Fuchs' conditions are incomplete, is quoted by both authors. It is

$$
(d y / d x)^{m}=y^{m+r}
$$

where $r$ and $m$ are coprime integers, $1 \leqslant r<m$. It has the solution $y=$ $\left(r\left(x_{0}-x\right) / m\right)^{-m / r}$, and has a movable branch point at which $y$ is infinite.

Fuchs' results were extended to the general case by Poincaré in his [1885], who showed that when the genus is greater than 1 the solution is an algebraic function of $z$. Poincaré's methods were based on his new theory of automorphic functions; indeed he expressed some regrets at the end of his paper that the new differential equations do not lead to a new class of transcendental functions. As such they were generally found to be more complicated than those of Picard, which were put forward in his Traité $d$ 'analyse of 1893 (vol. III, pp. 81-87). Picard's methods were based on the theory of birational equivalences of surfaces, and used Hurwitz' observation [1887] that a surface of genus greater than 1 admits only a finite number of birational self-transformations. Picard considered the equation $f\left(x, y, y^{\prime}\right)=0$ and a solution $y$ for which $y\left(x_{0}\right)=y_{0}$ and $y^{\prime}\left(x_{0}\right)=y_{0}^{\prime}$ for some point $x_{0}$. If analytic continuation of $y$ from $x_{0}$ to another nonsingular point $x_{1}$ produces a solution for which $y\left(x_{1}\right)=y_{1}$ and $y^{\prime}\left(x_{1}\right)=y_{1}^{\prime}$, then $f\left(x_{1}, y_{1}, y_{1}^{\prime}\right)=0$. Since $y$ and $y^{\prime}$ can each be considered as functions of $y_{0}$ and $y_{0}^{\prime}$, and conversely, therefore is a birational equivalence between $f\left(x_{0}, y_{0}, y_{0}^{\prime}\right)=0$ and $f\left(x_{1}, y_{1}, y_{1}^{\prime}\right)=0$ regarded as curves in $y$ and $y^{\prime}$. The correspondence is only birational because it may fail where either curve has a multiple point. Complicated, but essentially elementary, considerations of the holomorphic integrands on each curve gave Picard a necessary and sufficient set of conditions for any transformation of two curves to be a birational equivalence. From these he could deduce Poincaré's Theorem. In his [1888] Painleve showed that the first-order differential equations having all their singular points (i.e. poles) fixed coincides with Fuchs' class of equations. Then in his [1898, 1900 and 1902], Painlevé, again inspired by Picard, showed how these results can be extended, in modified form, to differential equations of higher order. In particular he gave his celebrated list of differential equations of the form $d^{2} y / d x^{2}=f(x, y, d y / d x)$, where $f$ is rational in $d y / d x$, algebraic in $y$, and analytic in $x$, which have only fixed poles and branch points and cannot be solved by quadratures nor reduced to linear equations. We have traced Fuchs' influence as far as the creation of Painlevé transcendents; it is time to turn back to Fuchs himself.

The first lesson Fuchs drew from his own observations on algebraic differential equations was that there were many such which did not have solutions that were analytic functions! In his [1885] he claimed that Jacobi had long since shown that $d x / d u=R(x)^{1 / 2}$, where $R(x)$ is a polynomial of degree greater than 4 , has solutions which are not analytic functions of $u$. This drew a pained response from his friend Casorati, who wrote an admirably clear paper [1886] precisely to persuade Fuchs, and others of his opinion, that

$$
z=\int_{0}^{Z} R(x, y) d x
$$


does define an analytic function $Z$ of $z$ whenever $R(x, y)=0$ is an algebraic function. Casorati showed that the function was, in the language of the time, infinitely many-valued. Fuchs' mistake lay in accepting uncritically Jacobi's definition of analytic. Jacobi had indeed shown that the values of $z$ for a given $Z$ cluster arbitrarily close together in the whole plane, and on that account he said $Z$ was not analytic as a function of $z$. Fuchs seems to have used Jacobi's terminology uncritically, although it conflicted with the contemporary sense of analytic (namely, locally expressible as a power series). For this he was privately criticized by Weierstrass, who wrote to his friend Sonya Kovalevskaya on 14 March 1885 that Fuchs confused taking a given value with getting arbitrarily close to a given value. "The values of $Z$ corresponding to one and the same value of $z$ make up a countable set concerning which Cantor has proved incontrovertibly, as I am overjoyed to say, that there are not only infinitely many values which are not in it, but that these form a set of higher cardinality." (quoted in Biermann [1966, p. 212]). In Cantor's terminology, the values of $z$ corresponding to a given $Z$ are everywhere dense. Casorati showed, however, that there is a perfectly good fundamental region for $Z$ as a function of $z$, but even when $R$ is hyperelliptic and of degree 5 the region has two sheets. This led him to form the corresponding Riemann surface for $Z$ which had infinitely many sheets. Casorati's work is discussed in detail in Bottazzini [1977, pp. 50-56], which also contains extracts from the Hermite-Casorati correspondence. Hermite wrote to Casorati (28 November 1885): "Besides, who would have disputed the dependence between the value of an integral and its limits. No one can raise any objection, neither to your reasoning nor its conclusion, concerning the entirely special law of this dependence which you have brought to light by employing Riemann surfaces." So it seems that Fuchs' mistake followed from his tenuous hold on the theory of Riemann surfaces and his neglect of the emerging Cantorian theory of point sets.

By 1885, the theory of functions had two foci: the great theory of complex analytic functions, and the rapidly developing theory of integration (Hawkins [1980] gives a vivid account of the journey from the Riemann integral to that of Lebesgue). The rich terrain between these two centres lay largely unexplored. Fuchs' remarks, however bungled, indicate a growing awareness of its importance. However, Fuchs himself did not enter the new territory, perhaps because he had got off to such a bad start, and instead turned back to old themes. In a series of four papers $[1886 \mathrm{~b}, 1887 \mathrm{a}, \mathrm{b}, \mathrm{c}]$ he returned to his Jacobi inversion problem and illuminated it with his theorem that a Riemann surface which admits an involution is birationally equivalent to a hyperelliptic one. This result is a special case of a theorem of Hurwitz, which says that any Riemann surface admitting an automorphism of period $n$ is birationally equivalent to one whose equation is $F\left(s^{n}, z\right)=0$. Hurwitz communicated this result by letter to Fuchs, who duly acknowledged it in his paper. Unhappily a priority dispute then arose, and Hurwitz attached a note to his paper alleging that Fuchs had slighted him (one suspects the hand of Klein). Fuchs replied in kind, and it seems Hurwitz thought better of it, for he withdrew the note when he republished the paper in the Mathematische Annalen in 1888, and would not allow it to be reprinted in the edition of his Werke (Hurwitz, Werke, I, 
editor's note p. 241). There can be little doubt that the discoveries were independent, and Hurwitz's paper is far more general.

But of greater interest is the last theme of Fuchs' work. He became increasingly concerned with the way the solutions to a differential equation depend on the coefficients. This is particularly a problem when a basis of solutions defined near one point is analytically continued to a second point where a basis of solutions is independently known. The problem is to find the matrix connecting the one system of solutions with the other. If this problem was solved, say for a linear equation, one would have an explicit representation of the monodromy group of the equation. In fact the problem is very difficult. Poincare discussed it in his [1884] in a way that none of his contemporaries seem to have liked because they never refer to it. Fuchs took up the case in which the monodromy matrices are independent of some of the coefficients of the equation, and was led to a system of first order partial differential equations which the solutions to the equation satisfy when considered as functions of those coefficients and the independent variable. In this way his work connected with his earlier study of hyperelliptic integrals as functions of a parameter, and also with contemporary work of Picard. The differential equations whose monodromy matrices are independent of a coefficient of the equation therefore have the property that their solutions are analytic functions of that coefficient as well as of the independent variable.

Fuchs' most successful student, Ludwig Schlesinger, took up this question in a series of papers, culminating in his [1912]. He called the problem of characterizing those differential equations that are independent of some of the coefficients in the equation the Fuchs problem, and showed how it was connected with the Riemann problem (find a differential equation of the Fuchsian type with specified monodromy matrices at given singular points). He had earlier 'solved' this problem by means of the so-called method of continuity and Poincaré's theory of zeta-Fuchsian functions [1905], but his method was justly criticized by Plemelj [1909], and he corrected some mistakes in his reply [1909]. At the same time Hilbert gave his discussion of the Riemann problem for differential equations in his paper [1905], and in 1906 Plemelj extended Hilbert's methods to the $n$th order case. For this reason, and because Hilbert stated the Riemann problem as the 21st in his list of mathematical problems, the problem is often called the Riemann-Hilbert problem. Unfortunately, a history of this fascinating problem would take us too far afield: the interested reader may consult Röhrl [1957] for a brief history and general solution, Forster [1977] for a simplified version in the special case where the equation is defined on a noncompact surface, and Katz [1976] for an algebraic-geometric reinterpretation of the problem.

Schlesinger in his [1912] gave explicitly the necessary and sufficient conditions the coefficients must satisfy if monodromy matrices are to be independent of the location of a singular point. These conditions were a system of partial differential equations, and he showed that they were solvable. The solutions were meromorphic functions with fixed poles and branch points, and Schlesinger gave them explicitly when the differential equation was of the second order. In the particular case of a second-order equation of the Fuchsian 
type with three fixed poles and one variable pole, $\tau$ say, the Schlesinger equations, as they are called, reduce to an ordinary differential equation for a function of $\tau$ which Fuchs' son Richard had already shown [1905] was one of Painlevé's types. (In fact, it is the most general of them.) However, Painlevé omitted it from his celebrated list, and it was first put there by Gambier [1906] in the course of a successful attempt to consummate Painlevé's work.

Nonlinear ordinary and partial differential equations with explicit solutions are rare, and apparently many of them share with Schlesinger's equation the property of representing integrability conditions for deformations of linear equations. Thus the 'isospectral' deformation of the Schrödinger operator is governed by the Korteweg-de Vries equation. A good starting point for modern work on such problems is Flaschka and Newell [1980], who also indicate some of the connections with physics that seem to arise.

Fuchs continued to work on mathematics right up to his death, which came when he was taking a walk on April 26, 1902. He was just nine days short of his 69th birthday. Biermann, whose study [1973] of Berlin University 1810-1920 is an essential starting point for any study of German mathematics in the period, quotes Fuchs' friend Koenigsberger as saying (p. 103) that Fuchs was "Indecisive and anxious, procrastinating and easily swayed, yet humorous and quite unselfishly kind". His colleagues seem to have liked him, and certainly something of his good qualities comes through in his photograph, which adorns the three volumes of his Collected Works, edited by Schlesinger and Richard Fuchs between 1904 and 1909.

Conclusion. Fuchs's career has invited easy simplifications. Some, notably his students Heffter and Schlesinger, have praised him for opening up a new mathematical province, a judgement shared by Hamburger and others at Berlin. On the other hand, Klein never missed an opportunity to speak slightingly of Fuchs' work, and as late as his Entwicklung der Mathematik [1926, p. 270] said of Fuchs and his students that they formed "a typical example of a strictly limited "School"' and that Fuchs "did not proceed further on paths taken by Riemann, but worked directly with the formulae in an elementary way". Both types of judgement reveal more about the speakers than their subject. Fuchs was indeed the leading Weierstrassian analyst of his day, and so naturally was regarded highly in Berlin. But his work was more fertile in other hands than his own, when it was connected to other traditions than those favoured in Berlin. Personal feelings aside, Fuchs' interests were indeed not central in Göttingen after the First World War, and partly as a result they have not recovered their former status in the world since. The monodromy problem seems only now to be capable of solution, and with the emerging theory of $p$-adic differential equations Fuchs's stock will probably rise. The Fuchsian class of differential equations is of interest to algebraic geometers, and the Picard-Fuchs equation seems an appropriate reminder of his interest in treating the coefficients of differential equations as parameters. But it would be idle to debate the justice of remembering Fuchs by things Fuchsian. Rather, Fuchs' career remains of interest because it shows clearly and dramatically how mathematical ideas are many-sided, and how many new ideas may be needed 
to solve a problem. There is an ironic truth in the assessment that Fuchs opened up a new province: repeatedly Fuchs pointed to problems in analysis that could best be solved by group theory. Denying himself this tool, which belonged to the younger generation, one might say that Fuchs could only stand like Moses and gaze upon the promised land. So our interest is not exclusively in Fuchs but also in his relationship to others, such as Klein, Hurwitz, and, above all, Poincaré. Finally, his career and the subsequent decline in his reputation dramatically illustrate how solutions in mathematics lead on to other problems, and how obstinately some problems force mathematicians to change what they regard as answers. The many interesting discoveries about the monodromy of differential equations made since Fuchs' time still fall short of answering the simple and direct questions he had in mind when investigating the relationship between ordinary linear differential equations and their integrals.

Appendix. The referee has kindly reminded me that two other papers by Riemann are of considerable interest in the historical study of differential equations. One is number XXI in the second edition of his Werke, and dates apparently from February 20, 1857. In this paper Riemann considered $n$ thorder differential equations all of whose monodromy matrices are diagonalizable, and showed how to write down the general form of such an equation when none of its solutions ever become "infinitely great to an infinitely great order", as he put it. Consequently, he obtained Fuchs' characterization of such an equation, although he presented it in the form it takes when $\infty$ is an ordinary point. The second paper consists of notes of some of Riemann's lectures from the winter semester 1858/59 taken by von Bezold, which are even more remarkable. Riemann first considered the integral form of the solutions to the hypergeometric equation, and then went on to look at quotients to the solutions of such equations and the inverse function to such quotients. He even showed how to recapture a differential equation with algebraic coefficients when given a function invariant under specified monodromy transformations; this is the origin of Hilbert's 21 st problem. He investigated in particular when the quotient is algebraic, and was led in this way to discover some results first published by Schwarz. The lectures conclude with a discussion of the modular function $k^{2}$ from this point of view; Riemann correctly describes its fundamental domain. Riemann may have seen this description in Gauss' Nachlass, which he was helping to edit, however, it is ironic to notice that the editorial team, led by Schering, eventually misunderstood this Gaussian fragment when they first published it in Gauss' Werke.

It is hard to assess the historical significance of these papers by Riemann. They have been well edited, perhaps one should say thoroughly edited, by Weber and Wirtinger, so one cannot be sure what has been done beyond the correction of mathematical mistakes in the manuscripts. The first paper was not published until 1876, the second not until 1902, and neither seems to have exerted any influence beforehand. But it is indisputable that Riemann saw some of the best ideas of Fuchs and Schwarz on differential equations before they did, although I am sure that their discoveries were made entirely independently. Wirtinger, in his lecture to the Heidelberg International Congress of 
1904 (Proceedings, p. 124) tells us that von Bezold was then at the commencement of his studies and could not appreciate Riemann's lectures at the time, but that he later brought them to the attention of his colleagues in Berlin. Fuchs in particular had a copy made for his own use in 1894. But von Bezold was a meteorologist and seems to have done nothing with Riemann's work himself. I have treated these questions in more detail in my [1983b] (to appear).

\section{BIBLIOGRAPHY}

F. Baldassari and B. Dwork (1979), On second order linear differential equations with algebraic solutions, Amer. J. Math. 101, 42-76.

K-R. Biermann (1966), Karl Weierstrass. Ausgewählte Aspekte seiner Biographie, J. Math. 223, 191-220.

(1973), Die Mathematik und ihre Dozenten an der Berliner Universität, 1810-1920.

Akademie Verlag, Berlin.

U. Bottazzini (1977), Le funzioni a periodi multipli nella corrispondenza tra Hermite e Casorati, Arch. Hist. Exact Sci. 18, 39-88.

F. Brioschi (1879), Sopra una classe di equazioni differenziali lineari del secondo ordine, Ann. Mat. (II) 9, 11-20 = Opere, vol. II, no. LXXIII (1901), 177-187.

C. Briot and J. Bouquet (1856a) Étude des fonctions $d$ 'une variable imaginaire, J. École Polytech. 21, 85-132.

(1856b), Recherches sur les propriétés des fonctions définies par des équations différentielles, ibid., 133-198.

F. Casorati (1886), Les lieux fondamentaux des fonctions inverses des intégrales abéliennes et en particulier les fonctions inverses des intégrales elliptiques de $2^{\text {ème }}$ et $3^{\text {ème }}$ espèce, Acta Math. 8, 360-386 = Opere, I, 1951, 239-265.

A. L. Cauchy (1835), Mémoire sur l'intégration des équations différentielles, Prague. (1840), Mémoire sur l'intégration des équations différentielles, Exercises d'Analyse et de Physique Mathématique. II, Paris = Oeuvres complètes, (2) vol. 11, 399-465.

A. Cayley (1883), On the Schwarzian derivative and the polyhedral functions, Trans. Cambridge Philos. Soc. 13, 5-68 = Collected mathematical papers, vol. 11, 148-216.

R. F. A. Clebsch (1864), Ueber die Anwendung der Abelschen Functionen in der Geometrie, J. Math. 63, 189-243.

R. F. A. Clebsch and P. Gordan (1866), Theorie der Abelschen Functionen, Teubner, Leipzig.

C. H. Clemens (1980), A scrapbook of complex curve theory, Plenum, New York and London.

R. Dedekind (1877), Schreiben an Herrn Borchardt über die Theorie der elliptischen Modulfunctionen, J. Math. 83, 265-292 = Werke, I, Chelsea, New York, 1969.

H. Flaschka and A. C. Newell (1980), Monodromy and spectrum-preserving deformations. I, Comm. Math. Phys. 76, 65-116.

O. Forster (1977), Riemannsche Flächen, Springer-Verlag, Berlin and New York.

F. G. Frobenius (1873a), Über die Integration der linearen Differentialgleichungen durch Reihen, J. Math. 76, 214-235 = Gesammelte Abhandlungen, I, 84-105, Springer-Verlag, Berlin and New York.

(1837b), Über den Begriff der Irreducibilität in der Theorie der linearen Differentialgleichungen, op. cit 76, 236-270 = Gesammelte Abhandlungen, I, 106-140.

(1875), Über die reguläre Integrale der linearen Differentialgleichungen, op. cit 80,

317-333 = Gesammelte Abhandlungen, I, 232-248.

L. I. Fuchs (1865), Zur Theorie der linearen Differentialgleichungen mit veränderlichen Coefficienten, Jahrsber. Gewerbeschule, Berlin, Ostern $=$ Werke, I, 111-158. (1866), ibid., J. Math. 66, 121-160 = Werke, I, 159-204.

(1868), ibid., Ergänzungen zu der im 66-sten Bande dieses Journal enthaltenen Abhandlung, J. Math. 68, 354-385 = Werke, I, 205-240.

(1870a), Die Periodicitätsmoduln der hyperelliptischen Integrale als Functionen eines

Parameters aufgefasst, J. Math. 71, 91-127 = Werke, I, 241-282.

(1870b), Uber eine rationale Verbindung der Periodicitätsmoduln der hyperelliptischen

Integrale, J. Math. 71, 128-136 = Werke, I, 283-294. 
(1870c), Bemerkungen zu der Abhandlungen “ ̈̈ber hypergeometrische Functionen nter Ordnung" in diesem Journal, 71, $316=\mathrm{J}$. Math. 72, 255-262 = Werke, I, 311-320.

(1871a), Über die Form der Argumente der Thetafunctionen und über die Bestimmung von $\theta(0, \ldots, 0)$ als function der Klassenmoduln, J. Math. 73, 305-323 = Werke, I, 321-342.

(187lb), Über die linearen Differentialgleichungen, welchen die Periodicitätsmoduln der Abelschen Integrale genügnen, und über verschiedene Arten von Differentialgleichungen für $\theta(0,0, \ldots, 0)$, J. Math. 73, 324-339 = Werke, I, 343-360.

(1875), Über die linearen Differentialgleichungen zweiter Ordnung, welche algebraische Integrale besitzen, und eine neue Anwendung der Invariantentheorie. . , Nachr. Königl., Gesellschaft Wiss., Göttingen, pp. 568-581, 612-613 = Werke, II, 1-10.

(1876a), Über die linearen Differentialgleichungen zweiter Ordnung, welche algebraische Integrale besitzen, und eine neue Anwendung der Invariantentheorie, J. Math. 81, 97-142 = Werke, II, $11-62$.

II, 63-66.

(1876b), Extrait d' une lettre adressée à M. Hermite, J. de Math. (3) 2, 158-160=Werke,

(1876c), Sur les équations différentielles linéaires du second ordre, Comptes Rendus 82, 1494-1497; ibid. 83, 46-47 = Werke, II, 67-72.

(1877a), Selbstanzeige der Abhandlung: “Über die linearen Differentialgleichungen zweiter Ordnung, welche algebraische Integrale besitzen und eine neue Anwendung der Invariantentheorie", Borchardts Journal 81, 97, sqq. Rep. Math., I, 1-9 = Werke, II, 73-86.

(1877b), Sur quelques propriétés des intégrales des équations différentielles, auxquelles satisfont les modules de périodicité des intégrales elliptiques des deux premières espèces. Extrait d'une lettre adressée à M. Hermite, J. Math. 83, 13-37 = Werke, II, 87-114.

(1878a), Über die linearen Differentialgleichungen zweiter Ordnung, welche algebraische Integrale besitzen. Zweite Abhandlung, J. Math. 85, 1-25 = Werke, II, 115-144.

(1877c), Extrait d'une lettre adressée à M. Hermite, Comptes Rendus 85, 947-950= Werke, II, 145-150.

(1878b), Über eine Klasse von Differentialgleichungen, welche durch Abelsche oder elliptische Functionen integrierbar sind. Nachr. Königl., Gesellschaft Wiss., Göttingen, pp. 19-32; Ann. Mat. (II) 9, 25-35 = Werke, II, 151-160.

(1878c), Sur les équations différentielles linéaires qui admettent des intégrales dont les différentielles logarithmiques sont des fonctions doublement périodiques. Extrait d'une lettre adressée à M. Hermite, J. de Math. (3) 4, 125-140 = Werke, II, 161-176.

(1879a), Selbstanzeige der Abhandlung; "Sur quelques propriétés des intégrales des équations différentielles, auxquelles satisfont les modules de périodicité des intégrales elliptiques des deux premières espèces. Extrait d'une lettre adressée à M. Hermite”, Borchardts Journal 83, 13, sqq. Rep. Math. 2, 235-240 = Werke, II, 177-184.

(1880a), Über eine Klasse von Functionen mehrerer Variabeln, welche durche Umkehrung der Integrale von Lösungen der linearen Differentialgleichungen mit rationalen Coefficienten entstehen, Nachr. Königl., Gesellschaft Wiss.,Göttingen, pp. 170-176 = Werke, II, 185-190.

(1880b), Über eine Klasse von Functionen mehrerer Variabeln, welche durch Umkehrung der Integrale von Lösungen der linearen Differentialgleichungen mit rationalen Coefficienten entstehen, J. Math. 89, 151-169 = Werke, II, 191-212.

(1880c), Sur une classe de fonctions de plusieurs variables tirées de l'inversion des intégrales de solutions des équations différentielles linéaires dont les coefficients sont des fonctions rationnelles. Extrait d' une lettre adressée à $M$. Hermite, Comptes Rendus 90, 678-680, 735-736= Werke, II, 213-218.

(1880d), Über die Functionen, welche durch Umkehrung der Integrale von Lösungen der linearen Differentialgleichungen entstehen, Nachr. Königl., Gesellschaft Wiss., Göttingen, pp. 445-453 = Werke, II, 219-224.

(1881a), A uszug aus einem Schreiben des Herrn L. Fuchs an C. W. Borchardt, J. Math. 90, 71-73 = Werke, II, 225-228.

(1880e), Sur les fonctions provenant de l'inversion des intégrales des solutions des équations différentielles linéaires. .., Bull. Sci. Math. Astronomi. (2) 4, 328-336 = Werke, II, 229-238.

(1881b), Über Functionen zweier Variabeln, welche durch Umkehrung der Integrale zweier gegebener Functionen entstehen. ., Abh. Königl., Gesellschaft Wiss., Göttingen 27, 1-39 = Werke, II, 239-274. 
(1881c), Sur les fonctions de deux variables qui naissent de l'inversion des intégrales de deux fonctions données. Extrait d' une lettre adressée à M. Hermite, Comptes Rendus 92, 1330-1331, 1401-1403 = Werke, II, 275-282.

(1881d), Sur une équation différentielle de la forme $f(u, d u / d z)=0$. Extrait d'une lettre adressée à M. Hermite, Comptes Rendus 93, 1063-1065 = Werke, II, 283-284.

(1882a), Über Functionen, welche durch lineare Substitutionen unverändert bleiben, Nachr. Konigl., Gesellschaft Wiss., Göttingen, pp. 81-84 = Werke, II, 285-288.

(1882b), Über lineare homogene Differentialgleichungen, zwischen deren Integralen homogene Relationen höheren als ersten Grades bestehen, Sitzungsberichte Gesellschaft Wiss., Königl. Preuss Akad. Wiss. Berlin, pp. 703-710 = Werke, II, 289-298.

(1882c), Über lineare homogene Differentialgleichungen, zwischen deren Integralen homogene Relation höheren als ersten Grades bestehen, Acta Math. 1, 321-362 = Werke, II, 299-340.

(1884a), Über Differentialgleichungen, deren Integrale feste Verzweigungspunkte besitzen, Sitzungsberichte Königl. Preuss Akad. Wiss. Berlin, pp. 699-710 = Werke, II, 355-368.

(1884b), Antrittsrede gehalten am 3. Juli 1884 in der öffentlichen Sitzung zur Feier des LEIBNIZtages, Sitzungsberichte Königl. Preuss Akad. Wiss. Berlin, pp. 744-747=Werke, II, 369-372.

(1884c), Über eine Form, in welche sich das allgemeine Integral einer Differentialgleichung erster Ordnung bringen lässt, wenn dasselbe algebraisch ist, Sitzungsberichte Königl. Preuss Akad. Wiss. Berlin, pp. 1171-1177 = Werke, II, 373-380.

(1885), Über den Charakter der Integrale von Differentialgleichungen zwischen complexen

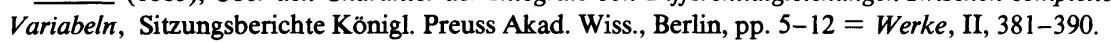

(1886a), Über die Werthe, welche die Integrale einer Differentialgleichung erster Ordnung in singulären Punkten annehmen können, Sitzungsberichte Königl. Preuss. Akad. Wiss., Berlin, pp. 279-300 = Werke, II, 391-416.

(1886b), Über diejenigen algebraischen Gebilde, welche eine Involution zulassen, Sitzungsberichte Konigl. Preuss Akad.Wiss., zu Berlin, pp. 797-804 = Werke, II, 417-426.

(1886c), Über eine Klasse linearer Differentialgleichungen zweiter Ordnung, J. Math. 100, 189-200 = Werke, II, 427-440.

(1887a), Über die Umkehrung von Functionen zweier Veränderlichen, Sitzungsberichte

Königl. Preuss Akad. Wiss., Berlin, pp. 99-108 = Werke, II, 441-452.

(1887b), Über einen Satz aus der Theorie der algebraischen Functionen und über eine Anwendung desselben auf die Differentialgleichungen zweiter Ordnung, Sitzungsberichte Königl. Preuss Akad. Wiss., Berlin, pp. 159-166 = Werke, II, 453-462.

(1887c), Bemerkungen zu einer Note des Herrn HURWITZ, enthalten in No. $6 \mathrm{Jahrg}$. 1887, S. 104 sqq. der Nachrichten, Nachr. Königl., Gesellschaft Wiss., Göttingen, pp. 345-347 = Werke, II, 463-466.

(1887d), Über Relationen zwischen den Integralen von Differentialgleichungen, Sitzungsberichte Königl. Preuss Akad. Wiss., Berlin, pp. 1077-1094 = Werke, II, 467-487.

(1888), Zur Theorie der linearen Differentialgleichungen, Sitzungsberichte Königl. Preuss Akad. Wiss., Berlin; Einleitung und No. 1-7, 1888, pp. 1115-1126: No. 8-15, 1888, pp. 1273-1290; No. 16-21, 1889, pp. 713-726; No. 22-31, 1890, pp. 21-38=Werke, III, 1-74.

(1890), Über algebraisch integrierbare lineare Differentialgleichungen, Sitzungsberichte Königl. Preuss Akad. Wiss., Berlin, pp. 469-488 = Werke, III, 81-98.

(1892), Über lineare Differentialgleichungen, welche von Parametern unabhängige Substitutionsgruppen besitzen, Sitzungsberichte Königl. Preuss Akad. Wiss., Berlin, pp. 157-176= Werke, III, 117-140.

(1893), Über lineare Differentialgleichungen, welche von Parametern unabhängige Substitutionsgruppen besitzen, Sitzungsberichte Königl. Preuss Akad. Wiss., Berlin, Einleitung und No. 1-4, 1893, pp. 975-988; No 5-8, 1894, S. 1117-1127 = Werke, III, 169-198.

(1904-1909), Gesammelte Mathematische Abhandlungen, 3 vols. (R. Fuchs and L.

Schlesinger, eds.), Berlin.

R. Fuchs (1905), Sur quelques équations différentielles linéaires du second ordre, Comptes Rendus 141, 555-558.

B. Gambier (1906), Sur les équations différentielles du second ordre et du premier degré dont l'intégrale générale est à points critiques fixes, Comptes Rendus 143, 741-743. 
C. F. Gauss (1812a), Disquisitiones generales circa seriem infinitam, Pars prior, Comm. Soc. Reg. Göttingen, II = Werke, III, 1876, 123-162.

(1812b), Determinatio seriei nostrae per aequationem differentialem secundi ordinis, ms. $=$ Werke, III, 207-230.

P. Gordan (1868), Beweis, dass jede Covariante und Invariante einer binären Form eine ganze Function mit numerischen coefficienten einer endlichen Anzhal solcher Formen ist, J. Math. 69, 323-354.

J. J. Gray (1981), Les trois suppléments au mémoire de Poincaré, écrit en 1880, sur les fonctions fuchsiennes et les équations différentielles, Comptes Rendus 293, 87-90. (1982), From the history of a simple group, Math. Intelligencer 4, 59-67.

(1983a), The three supplements to Poincaré's prize essay of 1880 on Fuchsian functions and differential equations, Arch. Internat. Hist. Sci. (to appear). , (1983b), Differential equations and group theory from Riemann to Poincaré (to appear).

G. H. Halphen (1884), Mémoire sur la réduction des équations différentielles linéaires aux formes intégrables, Mémoires présentées par divers savants à l'Académie des Sciences, vol. 28, pp. 1-260 = Oeuvres, III, 1921, 1-260.

(1888), Fonctions elliptiques. II, Paris.

M. Hamburger (1873), Bemerkungen über die Form der Integrale der linearen Differentialgleichungen mit veränderlichen Coefficienten, J. Math. 76, 113-125.

T. Hawkins (1977), Weierstrass and the theory of matrices, Arch. Hist. Exact Sci. 17, 119-163.

(1980), The origins of modern theories of integration, From the Calculus to Set Theory, 1630-1910, Duckworth, pp. 149-180.

C. Hermite (1877-1882), Sur quelques applications des fonctions elliptiques, Comptes Rendus 85-94, passim = Oeuvres, III, 266-418.

K. Heun (1889), Zur Theorie der Riemann'schen functionen zweiter Ordnung mit vier Verzweigunspunkten, Math. Ann. 33, 161-179.

D. Hilbert (1905), Über eine Anwendung der Integralgleichungen auf ein Problem der Funktionen Theorie, Verhandlungen des dritten internationalen Mathematiker-Kongresses 1904, Teubner, Leipzig, pp. 233-240.

A. Hurwitz (1887), Über diejenigen algebraische Gebilde, welche eindeutige Transformationen in sich zulassen, Nachr. Königl., Gesellschaft Wiss., Göttingen, pp. 85-107; reprinted in Werke (I), no. 12, pp. 241-259 without the appendix.

E. L. Ince (1926), Ordinary differential equations, Longmans.

C. Jordan (1870), Traité des substitutions et des équations algébriques, Paris.

(1876), Sur les équations du second ordre dont les intégrales sont algébriques, Comptes Rendus 82, 605-607 = Oeuvres, II, 1-4.

(1878), Mémoire sur les équations différentielles linéaires à intégrale algébrique, J. Math.

84, 89-215 = Oeuvres, II, 13-140.

(1880), Sur la détermination des groupes d'ordre fini contenus dans le groupe linéaire, Atti Accad. Napoli 8, no. 11 = Oeuvres, II, 177-218.

N. M. Katz (1976), An overview of Deligne's work on Hilbert's twenty-first problem, Mathematical Developments Arising from Hilbert Problems, Proc. Sympos. Pure Math., vol. 28, Amer. Math. Soc., Providence, R. I., pp. 537-557.

C. F. Klein (1875/76), Über binäre Formen mit linearen Transformationen in sich selbst, Math. Ann. 9, 183-208 = Gesammelte Mathematische Abhandlungen, II, no. LI, 275-301.

(1876), Über [algebraisch integrierbare] lineare Differentialgleichungen. I, Math. Ann. 11, 115-118 = Gesammelte Mathematische Abhandlungen, II, no. LII, 302-306. (1877), Review in Fortschritte der Mathematik of Fuchs[1876a].

(1878/79), Über die Transformationen der elliptischen Funktionen und die Auflösung der Gleichungen Fünften Grades, Math. Ann. 14, 111-172 = Gesammelte Mathematische Abhandlungen, II, no. LXXXII, 13-75.

(1921/23), Gesammelte Mathematische Abhandlungen, 3 vols, Springer-Verlag, Berlin.

(1926), Vorlesungen über die Entwicklung der Mathematik, im 19. Jahrhundert, reprinted by Chelsea, New York, 1967, 2 vols in 1 .

E. E. Kummer (1834), De generali quadam aequatione differentiali tertii orainis, Programme of the Liegnitz Gymnasium = J. Math. 100 (1887), 1-9 = Collected Papers, II, 33-39.

(1836), Über die hypergeometrische Reihe..., J. Math. 15, 39-83, 127-172 = Collected Papers, II, 75-166. 
G. Lamé (1845), Sur plusieurs théorémes d.'analyse démontrés par la theorie des surfaces orthogonales, Comptes Rendus 21, 112-117.

A. M. Legendre (1825), Traité des fonctions elliptiques et des intégrales Euleriennes, 3 vols., Paris.

M. Matsuda (1980), First order algebraic differential equations-a differential algebraic approach, Lecture Notes in Math., vol. 804, Springer-Verlag, Berlin and New York.

G. Mittag-Leffler (1880), Sur les équations différentielles linéaires à coefficients doublement périodiques, Comptes Rendus 90, 299-300.

E. Neuenschwander (1978a), The Casorati-Weierstrass theorem, Historia Math. 5, 139-166.

$1-89$.

$5, \overline{87-105}$. (1978b), Der Nachlass von Casorati (1835-1890) in Pavia, Arch. Hist. Exact Sci. 19, (1981), Studes in the history of complex function theory. II, Bull. Amer. Math. Soc. (N.S.)

P. Painlevé (1888), Sur les équations différentielles du premier ordre, Comptes Rendus 107, 221-224, 320-323, 724-726.

(1898), Sur la détermination explicite des équations différentielles du second ordre à points critiques fixes, Comptes Rendus 126, 1329-1332.

(1900), Sur les équations différentielles d'ordre quelconque à points critiques fixes, Comptes Rendus 130, 1112-1115.

(1902), Sur les équations différentielles du second ordre et d'ordre supérieur, dont

l'intégrale générale est uniforme, Acta Math. 25, 1-86.

E. Picard (1879), Sur une généralisation des fonctions periodiques et sur certaines équations différentielles linéaires, Comptes Rendus 89, 140-144.

(1880a), Sur une classe d'équations différentielles linéaires, Comptes Rendus 90, 128-131. (1880b), Sur les équations différentielles linéaires à coefficients doublement périodiques,

Comptes Rendus 90, 293-295.

(1893), Traité d'analyse. III, Paris.

J. Plemelj (1909), Über Schlesingers “Beweis" der Existenz Riemannscher Funktionenscharen mit gegebener Monodromiegruppe, Jahresber. Deutsch. Math.-Verein. 18, 15-20.

(1906), Neuer Existenzbeweis des Riemannschen Functionssystems mit gegebener Monodromiegruppe, Wien Akademische, Anzeiger.

H. Poincaré (1882a), Théorie des groupes Fuchsiens, Acta Math. 1, 1-62 = Oeuvres, II, 108-168. (1882b), Sur les fonctions Fuchsiennes, Acta Math. 1, 193-294 = Oeuvres, II, 169-257.

$300-401$. (1884), Sur les groupes des équations linéaires, Acta Math. 4, 201-311 = Oeuvres, II,

(1885) Sur un théorème de M. Fuchs, Acta Math. 7, 1-32 = Oeuvres. III, 4-31.

(1886), Sur les intégrales irreguliéres des équations linéaires, Acta Math. 8, 295-344=

Oeuvres, I, 290-332.

(1908), L'invention mathématique, Science et Methode, Paris, pp. 43-63.

(1916-1954), Oeuvres, 11 vols., Paris.

B. Riemann (1857a), Beiträge zur Theorie der durch Gauss'sche Reihe $F(\alpha, \beta, \gamma, x)$ darstellbaren Functionen, Königl. Gesellschaft Wiss., Göttingen = Werke, 67-83.

(1857b), Theorie der Abelschen Functionen, J. Math. 54, 115-155 = Werke. 88-144.

H. Röhrl (1957), Das Riemann-Hilbertsche Problem der Theorie der linearen Differentialgleichungen, Math. Ann. 133, 1-25.

L. Schlesinger (1905), Zur Theorie der linearen Differentialgleichungen im Anschlusse an das Riemannsche Problem, J. Math. 130, 26-46.

(1909), Bemerkung zum Kontinuitätsbeweise für die Lösbarkeit des Riemannschen Problems, Jahresber. Deutsch. Math.-Verein. 18, 21-25.

(1912), Über eine Klasse von Differentialsystemen beliebiger Ordung mit festen kritischen Punkten, J. Math. 141, 96-145.

A. Schlissel (1976), The development of asymptotic solutions of linear ordinary differential equations, 1817-1920, Arch. Hist. Exact Sci. 16, 307-378.

H. A. Schwarz (1872), Ueber diejenigen Fälle, in welchen die Gaussische hypergeometrische Reihe eine algebraische Function ihres vierten Elementes darstellt, J. Math. 75, 292-335 = Abhandlungen, II, 211-259.

J. Thomae (1870), Über die höheren hypergeometrischen Reihen, insbesondere über die Reihe..., Math. Ann. 2, 427-444. 
L. Thomé (1872), Zur Theorie der linearen Differentialgleichungen, J. Math. 74, 193-217.

H. Valentiner (1889), De endelige, Danish Acad. Publ. Ser. V, Vol. 6.

K. T. W. Weierstrass (1842), Definition analytischer functionen einer Veränderlichen vermittelst algebraischer Differentialgleichungen, $m s=$ Werke, I, 75-84.

(1856), Theorie der Abel'schen functionen, J. Math. 52, 285-339.

(1868), Zur Theorie der bilinearen und quadratischen Formen, Monatsberichte der Akademie der Wissenschaften zu Berlin, 310-338 = Werke, II, 19-44.

Faculty of Mathematics, Open University, Milton Keynes, MK7 6AA, BuckinghamSHIRE, ENGLAND

Current address: Department of Mathematics, Brandeis University, Waltham, Massachusetts 02254 